\title{
Entretien avec Christian Colin : mise en scène d' Othello, au T.E.P., nov. 1984
}

\section{Christian Colin}

\section{(2) OpenEdition \\ Journals}

Édition électronique

URL : http://journals.openedition.org/shakespeare/526

DOI : 10.4000/shakespeare.526

ISSN : 2271-6424

Éditeur

Société Française Shakespeare

Édition imprimée

Date de publication : 1 novembre 1989

Pagination : 153-162

Référence électronique

Christian Colin, «Entretien avec Christian Colin : mise en scène d'Othello, au T.E.P., nov. 1984 ", Actes des congrès de la Société française Shakespeare [En ligne], 6| 1989, mis en ligne le 01 janvier 2007, consulté le 30 avril 2019. URL : http://journals.openedition.org/shakespeare/526 ; DOI : 10.4000/ shakespeare. 526 


\section{SOCIETE FRANCAISE SHAKESPEARE}

\section{Actes du Congrès - 1984}

\section{LIEU ET TEMPS}

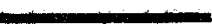

Directeur de la publication Jean FUZIER 


\section{Entretien avec Christian Colin Mise en scène d'Othello, au T.E.P., nov. 1984}

Sont présents trois membres de l'équipe qui joue Othello au Théâtre de l'Est Parisien : André Wilms (Othello), Christian Colin (metteur en scène) et Anne-Françoise Benhamou (assistante de mise en scène). Interviennent notamment dans le débat Margaret Jones Davies ( $M$. J-D.) Henri Suhamy (H. S.) Richard Marienstras (R.M.) Dominique Goy-Blanquet (D.G-B) et Michel Grivelet (M.G.).

Othello est la troisième mise en scène de Christian Colin, après Premier Amour de Beckett au Théâtre Gérard Philipe de Saint-Denis et La Peau Dure d'après Raymond Guérin. Comédien depuis très longtemps, il a joué notamment avec Bernard Sobel et l'équipe de Gennevilliers, avec Ariane Mnouchkine, Bruno Bayen. Il interprétait Ithamore dans Le Juif de Malte, mis en scène par Bernard Sobel, a joué deux fois dans La Tempête: Ariel, dans une mise en scène de B. Sobel, et Trinculo dans une mise en scène de François Marthouret. Surtout, il a joué Iago au Festival du Marais 1983 dans une mise en scène de Pierre Lamy, qui est lui-même actuellement sur le plateau et interprète le rôle du Duc et de Montano (T.E.P.). 
André Wilms a travaillé neuf ans au Théâtre National de Strasbourg avec Jean-Pierre Vincent et André Engel. Il a interprété récemment le rôle de Damis dans Tartuffe, mis en scène par Jacques Lassalle.

Q. La première question qui s'impose quand on a décidé de monter Othello est la question de la distribution: prendre ou non un Noir. Là, en l'occurence, c'est un blanc qui n'est pas passé au brou de noix, qui n'est pas grimé en noir, mais qui est grimé en gris-cendré. Comment êtes-vous parvenus à ce griscendré ? Cela me fait penser aux hommes gris du désert, mais cela évoque surtout une force d'altérité totale, de radicalisation de l'autre.

C.C. La question qui nous occupait c'était le discours sur l'étranger. Au départ, j'avais presque envie d'avoir un homme sans visage, en référence à un texte de Beckett, L'Innommable. Dans le travail, c'est très difficile de demander cela à un acteur. Le problème de la couleur se posait. Pendant un moment, je pensais à quelque chose de terreux, d'argileux, à un visage abîmé, déformé par une blessure. Le gris est intervenu très simplement, c'est une couleur qui me parle beaucoup et qui en plus se théâtralise, qui ne rend compte de rien d'autre que de la couleur. Il reste cependant au fond de moi un Othello sans visage. Dans la mise en scène, on essaie de suivre cette voie: pendant tout le début, on ne discerne pas bien Othello, mais comme André est un acteur qui joue beaucoup avec son visage, le fragmenter plus que cela me paraissait sacrilège.

Q. Et les cheveux roux ?

C.C. Ce sont les siens qui ressortaient roux avec le maquillage et la lumière.

J'avais lu dans Libération une chose qui m'avait intéressée sur un chanteur nègre albinos, suivant laquelle les nègres albinos étaient considérés en Afrique comme d'étranges personnages, pratiquement des sorciers, des personnes sacrées. Aussi pendant un moment, on a pensé à la dépigmentation, ce qui est très difficile à rendre par le maquillage. Et il aurait fallu utiliser d'autres éléments qui auraient pu être génants dans le jeu parce 
qu'André transpire beaucoup et que c'est exténuant d'utiliser des prothèses. On avait aussi pensé à des déformations du visage.

A.W. Orson Welles s'était mis des prothèses, mais ce qui est faisable au cinéma n'est pas faisable au théâtre sur une durée plus longue. Par contre, Laurence Olivier avait joué le Noir à fond : il avait vécu un an avec un Noir, et il s'était peint le corps entièrement en noir, et il avait imité la gestuelle noire. Dans L'Habilleur, Albert Finney se fait juste un masque. Donc, il y a toutes sortes de possibilités et une longue tradition.

Q. $\quad$ Et on peut aussi prendre un acteur noir?

C.C. Cela n'a pas pu se faire. Et d'une certaine façon, j'en suis très heureux parce que cela m'a permis de travailler d'une façon plus ouverte sur le personnage d'Othello.

Q. Un acteur noir t'aurait paru une limitation des propos d'Othello?

C.C. Oui, c'est l'œuvre d'un auteur blanc. C'est important de le dire, car il ne faut jamais perdre de vue l'écrivain. Le rapport à l'étranger me paraît beaucoup plus intéressant parce qu'il dépasse le problème des races.

Q. Le soir où j'ai vu la pièce, je ne l'ai pas aimée. Et ensuite, quand je me suis retrouvée seule, j’ai été frappée par l'espèce de pouvoir qu'elle a eu sur moi. Le mot qui m'est venu à l'esprit pour la caractériser est "neutralisation". Toute la violence d'Othello jusqu'à la dernière scène où il crie était en fait complètement neutralisée. On ne la ressent pas au début, c'est au moment où il crie que la violence apparaît. Voilà pourquoi j’ai aimé la mise en scène. Est-ce Shakespeare ou non? C'est une autre question. Beaucoup de gens ont été frappés par l'hystérie presque continuelle de Desdémone. En relisant le texte, je me suis aperçue que Shakespeare parlait à ce moment-là de * free speech ". L'hystérie est bien un langage libre. En fait, on ne lui reproche qu'une chose : c'est d'être ignorante.

Mais comment faut-il interpréter ce cadavre entouré de bandelettes qui fait penser au Locataire de Roman Polanski? Et 
à la fin ce bandeau qui se déroule à perpétuité ? J'aimerais savoir si tout le monde trouve une réponse à cette question que vous avez posée à la fin de la pièce.

C.C. Je peux répondre de façon très simple sur le personnage de l'homme aux bandelettes. J'ai déjà dit que j'aurais aimé qu'Othello soit bandé, qu'il soit sans visage. Je suis toujours touché par.... la bande velpo. Je voudrais savoir ce qu'il y a dessous. J'ai donc mis des bandes sur un visage, pour qu'on ne voie pas ce qu'il y a en dessous. Quand on crée une image, c'est pour susciter les questions. On a déjà donné beaucoup d'interprétations différentes: certains ont dit que c'était la dernière bande. André dit que c'est une zébrure, un drain.

Q. Quand on a un peu voyagé cela fait penser au départ de Barcelone en bateau : ceux qui restent sur le rivage lancent un rouleau de papier.

Dans le spectacle, le grotesque est présent de façon évidente dans le jeu - ainsi que la dérision. Quelle fonction ont ces éléments?

A.W. En tant qu'acteur, on sent toujours une dimension grotesque évidente.

Q. Mais dans le personnage d'Othello, elle n'est pas évidente dans l'écriture du rôle.

A.W. Si, je suis formel. Toute la scène avec Iago devant la baie vitrée est une scène de pur grotesque : "Je vais l'assassiner, je vais la tuer" "Oh, une femme si douce, une femme si fine, une femme si belle ".... Et la scène où il se cache est une pure scène de comédie. C'est le contraire de Tartuffe qui entend mais ne voit pas. Là, il voit mais n'entend pas. A mon avis, l'écriture même de cette scène est grotesque.

Q. Certaines scènes peuvent être écrites potentiellement de façon comique ou tragique, mais ici, le comique réside-t-il dans l'écriture, je n'en suis pas du tout certain.

C.C. La situation l'est, en tous cas.

Q. Elle n'est pas traitée comme telle, me semble-t-il. 
C.C. Je suis ému par le grotesque. J'aime beaucoup l'aspect Bokassa du rôle, avec ses dorures...

A.W. On m'a beaucoup reproché d'être petit. Dans la tête des gens, Othello est grand. Comme Clavier est un géant, je paraissais petit. Le Quotidien de Paris a même écrit: "Othello joué par un nabot". Or Othello est un héros, mais Shakespeare le met dans une sale histoire dès le début

Q. Cela ne détruit pas l'idée que ce soit un héros.

C.C. Quand même, il n'est pas gâté par Shakespeare.

A.F.B. Le héros part à la guerre, on le sait dans le texte, mais on ne le voit pas dans l'action. On nous le dit comme on nous raconte le passé d'Othello, comment il a séduit Desdémone. Ce que l'on voit ensuite, c'est son départ pour le combat. Ensuite l'armée turque est engloutie, un peu comme l'Invincible Armada qui s'est plus ou moins engloutie toute seule. Verdi, lui, avait demandé à son librettiste de faire revenir les Turcs, quand Othello part pour la guerre, pour qu'il y ait une scène de vivats. Il y a quelque chose qui manque en jouant cette scène. On aurait envie d'un héros victorieux, et pourtant le héros arrive le dernier, il commence à adresser des discours à Desdémone, et annonce que tous les Turcs sont noyés, ce que tout le monde sait depuis au moins vingt minutes. La dimension grotesque est toujours présente dans les grands personnages de Shakespeare. Un article de William Auden dit que Iago reprend toute la fonction critique du clown. Je crois que le grotesque est inscrit dans la pièce, mais je ne parlerais pas de dérision.

H.S. Il ne faut pas confondre les catégories du burlesque, du grotesque et du comique. Le contre-point entre le comique et le tragique est un fait shakespearien bien connu. Ce n'est pas parce qu'il y a une scène de comique ou de démythification comme dans Roméo et Juliette ou dans Othello qu'il faut gommer systématiquement la dimension héroïque ou grandiose. C'est un fait que l'on a le fameux "Exultate..." au début de l'Otello de Verdi qui semble affirmer la dimension héroïque du personnage, alors que, dans la pièce de Shakespeare, il n'a pas 
l'occasion, si l'on s'en tient simplement au texte, de tirer de coups de feu. Mais c'est tout au long de la pièce que son passé héroïque est commenté, rappelé, y compris par Iago. Iago, se lance dans de véritables discours où il parle d'Othello comme d'un général extraordinaire, et jusqu'à la fin, Cassio se réfère constamment à Othello comme à un homme de guerre particulièrement digne de respect et d'admiration. Le texte rappelle constamment la dimension héroïque du personnage.

R.M. On ne sortira pas de l'affaire en disant qu'on interprète Iago ou Othello de telle ou telle manière. Il y a un parti-pris général dans la mise en scène qui transpose la pièce au XIX ${ }^{\mathrm{e}}$ siècle, ou quelque chose qui ressemble au XIX ${ }^{\mathrm{e}}$.

C.C. Les costumes seraient plutôt 1940-50...

R.M. La dérision ne provient pas du texte mais du cadre dans lequel on l'a placé. C'est par conséquent un parti-pris de mise en scène.

Ma deuxième remarque porte sur l'héroïsme. Othello ne tient pas à faire état de sa valeur, ce qui ne veut pas dire que ce n'est pas un héros. C'est "un héros au repos", si je puis dire. Il y a toujours chez Shakespeare des personnages héroïques qui sont de véritables héros (dans les Henry $I V$, par exemple), et il y a des personnages qui en font la dérision. Mais pour en faire la dérision, il faut que le héros existe de son côté, sinon il n'y aurait pas de quoi se moquer, ni qui moquer. Au lieu de mettre la dérision dans la bouche du personnage qui diminue le héros, c'est la mise en scène qui le diminue. Vous enlevez une partie de la fonction du personnage négateur. Que devient-il, puisque c'est vous qui imposez la dérision? Quand le personnage se suicide, vous lui envoyez un geste de pitié un peu méprisant. Quand vous accueillez le vaisseau d'Othello avec un personnage sous un parapluie: "une voile, une voile", ou un fourbe qu'on assassine avec "au secours, au secours ", ce n'est que de la mise en scène, et les spectateurs ont été à juste titre surpris. 
C.C. Je n'est pas conscience de travailler dans la dérision. Je relisais un petit texte de Foreman qui dit: "je suis très surpris quand on n'aime pas mes spectacles". Tel que je le mets en scène, j'ai l'impression de traiter Othello de façon tragique.

Pour répondre à la question sur le $\mathrm{XIX}^{\mathrm{e}}$, en tant que metteur en scène, je ne peux pas faire autrement que de parler de ce qui me touche de près. J'ai une mémoire photographique du $\mathrm{XIX}^{\mathrm{e}}$ siècle que je veux représenter. Peut-être l'ai-je choisi à cause des thèses de Gobineau, parce que c'est une grande période de colonialisme. Mais il y a aussi des choses qui sont beaucoup plus proches de nous, qui proviennent des années 50 . Le spectacle est composé de morceaux de ma mémoire.

Je ne pense pas que la mort d'Othello soit un suicide, c'est un deuxième meurtre. Othello se tue devant Lodovico et les autres, mais j'ai voulu laisser Othello seul sur scène car je crois qu'il est, depuis le départ, un personnage abandonné, en sursis. Il commet donc un deuxième meurtre: il tue le monstre. Cet acte m'apparait comme dérisoire.

Les deux personnages que j'ai créés avec des bouts d'éléments ont pour moi une existence propre. Le personnage d'" une voile, une voile" m'a fait penser à des personnages beckettiens, des personnages qui ne parlent pas objectivement, qui n'ont rien à voir avec l'histoire, mais qui ont un rapport intermédiaire. Parfois, ce sont les personnages qui me fascinent le plus. Il est vrai qu'ils peuvent produire de la dérision.

Q. C'est une dérision qui peut être prise comme une atteinte au sérieux, au tragique.

C.C. Que cela porte atteinte au sérieux, c'est possible. Mais la sérieuse mise en scène d'Orson Welles n'est pas sérieuse; par exemple, il coupe, il intervertit, il prend cinq Desdémone, il fait un massacre, mais son film sert quand même de référence.

D.G.B. A mon avis, la pièce dramatise le mouvement de chute d'un héros. Chez vous, cette chute est jouée dès le début, c'est pour cela qu'il vous manque quelque chose.

C.C. En effet, je crois lire qu'à Venise, tout est déjà joué pour 
Othello. Je joue effectivement : « tout est perdu ». La seule porte de sortie d'Othello, si l'on peut dire, c'est Desdémone. C'est elle qui peut le sauver, en tant qu'homme, dans son identité. Et Cassio qui était toujours à l'arrière-plan se met brusquement en avant à l'arrivée à Chypre.

D.G.B. Cela ne se voit pas très bien dans la mise en scène. Si l'on n'a pas lu le programme, je me demande si l'on peut se douter que c'est lui qui recueille les fruits de cette triste histoire.

M.J.D. Pourquoi dit-on dans le programme que c'est Cassio le Machiavel, et non pas Iago ?

C.C. Iago ne commence pas la pièce. Il est totalement ignorant d'une série de choses. Et justement, dans une courte scène, on fait sortir Othello pour que Iago, sur scène, puisse poser la question (dont Cassio connaît la réponse) : "Que fait Othello ici ? ", "Il est marié ? ", "A qui ? ".

R.M. Ce n'est pas la seule scène de ce genre dans Shakespeare. Mais Iago en dit tellement plus, c'est lui qui parle le plus dans la pièce. Si Shakespeare avait voulu que Cassio soit un Machiavel, il l'aurait écrit de façon éclatante. Mais il est impossible de contourner Iago. C'est lui le Machiavel de la pièce et cela ressort même de votre mise en scène.

C.C. Je ne pense pas que Iago soit le Machiavel qu'on croit car il ne se bâtit sur rien, il n'a pas de projet réél : ce n'est pas lui qui ramasse le mouchoir, c'est sa femme.

Q. Dans l'écriture du rôle de Iago, il y a une part d'improvisation qui est réelle, et qui ressort dans la mise en scène, ou il est pas traité comme un "stéréotype " de traître. Cela lui donne une totale crédibilité par rapport aux autres. Il apparait un peu comme un gardien de musée, peut-être à cause de son costume, plus mû par une sorte de ressentiment que par un calcul machiavelique déjà présent en totalité dès le départ. Ce choix interprétatif est très sensible.

M.G. Dans la pièce, le couple Iago-Othello est, un couple antagoniste, et en même temps complice. Non seulement Iago veut la 
peau d'Othello, mais il lui est intimement lié par une sorte d'honnêteté. Franc, il dit ce qu'il pense. Il ne croit pas aux sentiments, il ne croit qu'à l'intérêt, l'amour n'existe pas, il n'y a que des faux-semblants. Il est parfaitement logique. Il sait que les hommes sont gouvernés par les intérêts et par les appétits. C'est clair. Il croit qu'Othello l'a trompé, et il veut sa peau aussi pour des questions de carrière. Iago est un militaire qui veut faire carrière, qui s'en sent capable. Si la pièce est bien montée, cela doit se sentir depuis le début. Même si ce n'est pas la guerre, c'est un monde de militaires, et de haines qui peuvent s'immiscer entre hommes qui vivent ensemble. Le fait que Iago improvise au fur et à mesure, voilà ce qui paraît génial de la part de Shakespeare. La haine se trouve toujours des stratégies pour s'assouvir.

Q. C'est un spectacle qui est très bavard, il dure trois heures, mais on ressent toujours une émotion glacée. Je ne l'ai supporté que parce que j'ai trouvé Iago très vraisemblable - naturaliste dans son jeu, par rapport à des personnages très distanciés.

C.C. La volonté de tenir la bride à l'émotion est très présente. Personnellement, je suis ému de voir ces rapports cruels entre les différents êtres. Je n'ai pas voulu faire de Iago vraiment un méchant. Je ne sais pas s'il l'est ou non. Il est vrai que c'est un personnage terrifiant, mais ils le sont tous, même s'ils sont ensuite envahis de culpabilité.

Q. Le début donne déjà une image cauchemardesque, avec les visages blêmes, les éclairages réduits. C'est peut-être là que le projet de mise en scène est le plus sensible, le plus fort, le plus présent.

M.J.D. Etait-ce nécessaire de partager le visage d'Émilie à la fin, et de la couvrir de pustules comme une lépreuse? Vous avez voulu montrer les deux côtés du personnage, mais à la fin, elle reconnaît sa faute, elle devrait être moins lépreuse qu'au début.

C.C. La pièce fait beaucoup état de corps qui s'abîment, qui se défont. J'avais l'impression qu'on ne le montrait jamais assez, alors que c'est un thème qui a l'air d'être très cher à Shakespeare. 
Q. Ce n'est pas Beckett qui transparait?

C.C. Non, les gens ne meurent pas vraiment chez Beckett. Là, ils meurent, sur la scène même.

Q. Votre position est plus désespérée, si ce n'est beckettienne, que tragique. Les personnages sont tous terrifiants, et enfermés dans une sorte de désespoir à la Beckett.

C.C. Je ne trouve pas cela tout à fait triste d'être désespéré. Peutêtre que je commets des choses terribles sur l'œuvre, mais j'ai eu envie de mettre en scène Othello particulièrement en ce moment, car je trouve que Iago se promène un peu trop dans les têtes. J'avais aussi envie de montrer qu'il y plus d'un Othello qui se suicide, qui commet un double meurtre. 